\title{
Response to the Comment on: Occipital C1-C2 Neuromodulation Decreases Body Mass and Fat Stores and Modifies Activity of the Autonomic Nervous System in Morbidly Obese Patients—a Pilot Study
}

\author{
Jacek Sobocki $^{1}$
}

Published online: 30 November 2015

(C) Springer Science+Business Media New York 2015

Thank you for the interest and comment of my paper. However, I would like to clarify some misunderstandings of author's comment.

First: C1-C2 occipital stimulation is far not the same as vagal neurostimulation (VNS). Actually, the only similarity is neuromodulation. The site of stimulation, stimulation time and schedule, type of fibers and nerves roots of CNS access, first and second neurons nuclei, and finally mechanism of action of these two approaches are different should not be mistaken. It is postulated that the former acts via nucleus raphe and the last acts via nucleus tractus solitarius (NTS).
Second: Authors suggest that VNS was shown to be effective in weight loss. In fact, this effect is clinically insignificant.

Third: Percutaneous stimulation of the anterior branch of the vagus nerve should not be mistaken with $\mathrm{C} 1-\mathrm{C} 2$ stimulation as well.

Morbid obesity is a chronic condition and thus requires chronic treatment. Chronic means here several hours every day for several weeks and months. Percutaneous electrodes might appear to be difficult to maintain for longer period of time. In contrary, preprogrammed implanted system working without necessity of engagement of the patient is supposed to be more comfortable and effective. A comparative study is mandatory to answer this question.

Jacek Sobocki

sobockij@gmail.com

1 Department of General Surgery and Clinical Nutrition, Medical

University of Warsaw, Warsaw, Poland 